\title{
Proceeding
}

Supplementary Issue: Summer Conferences of Sports Science. Costa Blanca Sports Science Events, 25-26 September 2020. Alicante, Spain.

\section{Acute effect on working memory in trained university students: A continuous exercise in different environments}

\author{
SALVADOR BAENA-MORALES ${ }^{1}$, HONORATO MORENTE-ORIA² , DANIEL RAMOS-PÉREZ², \\ FRANCISCO TOMÁS GONZÁLEZ-FERNÁNDEZ3 \\ ${ }^{1}$ Department of General and Specific Didactic, Faculty of Education, University of Alicante, Spain \\ 2Didactic of Languages, Arts and Sport's Department, University of Malaga, Spain \\ ${ }^{3}$ Department of Physical Activity and Sports Sciences, Pontifical University of Comillas, Mallorca, Spain
}

\begin{abstract}
The associated benefits of physical exercise on cognition have been studied in terms of different variables. These benefits in natural environments have been studied during the last decades, and several investigations have shown contradictory results, it could be explained by the diversity of methodological variables that influence the results. The present study aims to analyse the acute effect of a $10 \mathrm{~km}$ resistance test in two environments (natural and artificial) on working memory, evaluated with the Digit Span Test. Thirteen highly trained University Students exceeded the inclusion criteria for the study, the methodological recommendations found in previous studies were implemented. The physical exercise intervention was running 10 kilometres at a rate of between $4.00 \mathrm{~min} / \mathrm{km}$ and $4.10 \mathrm{~min} / \mathrm{km}$. This exercise was done in two circuits, in an artificial and natural environment. The subjective perception of effort and the control over the difficulty of the two circuits were controlled to confirm the same level of intensity. One-way ANOVA with Pre-Artificial Environment Condition $(0.23 \pm 0.16)$ and Post Artificial Environment Condition (0.94 \pm 0.03$)$ ] revealed a significant main effect of environment condition, $t(13)=2.14, p$ $<.05, d=0.62$. The results obtained showed how the natural environment produced significant improvements however, the artificial environment improved the results in the cognitive test, although not significantly. The results could be explained by the theory of restoration of attention that natural environments represent, confirming the hypothesis that the performance of physical exercise in natural environments produces an additional effect on working memory.

Keywords: Working memory; University students; Cognition; Physical exercise.

\section{Cite this article as:}

Baena-Morales, S., Morente-Oria, H., Ramos-Pérez, D., \& González-Fernández, F.T. (2020). Acute effect on working memory in trained university students: A continuous exercise in different environments. Journal of Human Sport and Exercise, 15(4proc), S1071-S1080. doi:https://doi.org/10.14198/jhse.2020.15.Proc4.09

Corresponding author. Didactic of Languages, Arts and Sport's Department, Faculty of Education, University of Málaga. Boulevard Louis Pasteur 25, 29010 Málaga. Spain. https://orcid.org/0000-0003-2335-1156

E-mail: hono@uma.es

Abstract submitted to: Spring Conferences of Sports Science. Costa Blanca Sports Science Events, 19-20 June 2020. Alicante, Spain. Communication highlighted as "Best Communication Award".

JOURNAL OF HUMAN SPORT \& EXERCISE ISSN 1988-5202

(c) Faculty of Education. University of Alicante

doi:10.14198/jhse.2020.15.Proc4.09
\end{abstract}




\section{INTRODUCTION}

Recent literature there has been a progressive increase in interest relating to the performance of physical exercise to different cognitive variables in all types of populations' improvements. These investigations have succeeded in demonstrating how physiological phenomena associated with an exercise produce progresses in memory performance (Coles \& Tomporowski, 2008; Tomporowski \& Ganio, 2006), attention (Harte \& Eifer, 1995), or surveillance (Naveen et al., 2017; Sanabria et al., 2019), among other variables related to cognition. That is why it seems to consolidate an important theoretical basis that justifies the relationship between cognitive performance and the performance of a certain physical effort. However, the diversity of variables that can condition the consolidation of this relationship remains an aspect that should be studied in depth. Within these variables, it has been possible to observe how the intensity, volume or type of physical exercise, as well as the level of the physical condition of the person, have consolidated as factors that modify cognitive performance (Costigan et al., 2016; González-Fernandez et al., 2020; Sanabria et al., 2019; Singh et al., 2019). In addition to these variables, the environment where physical exercise is performed has also been shown as a conditioning variable, since there are various works that support the idea that natural environments could help improve performance in cognitive assessment tests to a greater extent than artificial environments (Berman et al., 2008; Bratman et al., 2012; Rogerson et al., 2016). As mentioned above, the effects of the present natural environment on cognition have been highly studied. The findings found in the literature suggest how nature itself produces improvements in concentration, sense of well-being, attention and working memory (Bodin \& Hartig, 2003; Bratman et al., 2015; Lee \& Maheswaran, 2011; Pretty, 2004). Consequently, there seems to be some consensus when explaining that the cognitive improvement produced in natural environments, performing an activity, whether physical or not, is justified with the theory of restoration of care (Kaplan, 1995). This theory points out that urban environments can cause greater cognitive demands than natural environments, depleting attentional resources and, therefore, decreasing cognitive performance in tasks that require selective attention or working memory (Hartig et al., 2003; Kaplan, 1993). In addition, Bratman et al., (2015) emphasize how natural environments cause people a kind of "fascination" that helps replenish attention. This is why better performance is assumed in tests that measure working memory and attention after having remained in a natural environment.

These antecedents, that positively relate the binomial physical exercise in a natural environment with cognitive performance, have led researchers to focus efforts on analysing a possible "multiplier effect" of uniting both variables. Therefore, the set of physical exercise in natural environments (PEN), known in the literature as green exercise, has been highly studied through different systematic reviews and meta-analyses (Bowler et al., 2010; Lahart et al., 2019; Thompson Coon et al., 2011). However, Lahart et al., (2019) after a meta-analytical process, question the additional effect of performing PEN on physical exercise in artificial environments (PEA), arguing that there is no clear conception in the literature about it, being able to find a diversity of results, both for and against, around this theory.

The lack of consensus found in previous studies could be explained by the diversity of variables that condition the results (Baena-Morales et al., In Press). In addition to the aforementioned effects of the environment, they are joined by others such as the intensity of the physical activity or exercise performed, the duration of the stimulus, the study population, or even the cognitive test performed (Baena-Morales et al., 2020), which may complicate the comparison between some investigations and others. As indicated, the use of different cognitive assessment tests is one of the reasons for the disagreement between previous research (BaenaMorales et al., 2020). One of the tests that has been most widely used to compare the effect of PEN and ADP is the Digit Span Test. This test has also been widely used to assess verbal memory, attention or working memory (Otroski-Solís \& Lozano, 2006). Furthermore, it has been assumed that digit span tasks not 
only measure attention within the context of formal assessment, but also generalize to aspects of everyday life (Groth-Marnat \& Baker, 2003). This test can be performed in two ways, by recalling a sequence of digits forward and by recalling the sequence of digits backward. The backward sequence is more complex as it requires more mental control, because the person has to manipulate the information and retrieve it from his memory (Sohlberg \& Mateer, 1989). Therefore, DST allows the evaluation of the attention control system responsible for the selection of strategies, control and coordination of cognitive processes (Otroski-Solís \& Lozano, 2006).

In addition to this factor, there appear to be methodological shortcomings in the research aimed at comparing PEN with PEA (Lahart et al., 2019). For example, some studies that have analysed the additional effect of PEA on working memory have not included in their design the figure of a control group, nor have they checked whether the two experimental groups performed the physical exercise at the same intensity or supervised whether there was an excessive cognitive requirement during the performance of the task, as could involve talking to other participants or excessive traffic (Baena et al., 2020). Also, in previous literature studies have mainly evaluated the performance of various exercises, such as walking (Bailey et al., 2018; Berman et al., 2008; Bratman et al., 2015; de Brito et al., 2019; Fuegen \& Breitenbecher, 2018), cycling (Rogerson et al., 2016) or running (Bodin \& Hartig, 2003; Rogerson \& Barton, 2015). Finally, variables such as the duration of the exercise or the intensity of the exercise are other variables that can condition the results on the working memory. It has been observed that the acute interventions that carried out foot race were, mainly, at a moderate and light intensity, and with a maximum duration of 60 minutes (Bodin \& Hartig, 2003; Rogerson \& Barton, 2015), being only the study of Bodin \& Hartig (2003) the only one who examined the effect of physical exercise on working memory in non-professional runners.

Also, there has been a significant lack of consensus in the literature on a possible additional effect of PEN on PEA. Besides, since exercise has the acute effect, studies should be considered with a sample that has a similar level of physical condition, thus minimizing a possible polluting effect of the intensity or volume of the exercise performed. For all these reasons, the present study has been based on the deficiencies found in the previous literature to implement a research design whose main purpose is to avoid the weaknesses of previous research.

For these reason, this research's objective is to compare the results obtained by highly trained university students in a test of attention and working memory such as DST, after a physical exercise intervention in two different environmental contexts. According to the related literature, the hypothesis is that there will be no significant differences between the intervention in the natural environment and that performed in a stable environment. The results obtained will help to clarify to what extent variables such as the level of physical condition or the environment can exhaust the capacity of care or benefit and restore it.

\section{METHOD}

\section{Participants}

A total of 13 highly trained university students participated in the study (age $=28.4 \pm 3.25$ ). These inclusion criteria were used: at least five years of experience in athletics, a weekly training volume of more than 30 kilometres and an approximate performance of 40 minutes over a distance of 10,000 meters. All participants signed an informed consent form that followed the ethical guidelines proposed in the Helsinki Declaration. 


\section{Design and procedure}

A pre-post randomized crossover design was carried out, dividing the study into three main sessions.

In the first session, the subjects attended an informative meeting where the objectives of the study were explained, also, the procedure and tests, as the conditions to participate. To avoid that several variants could contaminate the results, the cognitive test was presented and carried out previously. They were also informed of the importance of carrying out the experiment avoiding conversations with other participants in the study and were informed of the route to be taken. The subjects were instructed not to perform any strenuous training for at least 72 hours before the execution of the resistance tests. Additionally, they were instructed not to drink stimulant beverages, reminding through text messages the importance of being well hydrated at the time of the tests and taking care to respect the usual time of training of each subject. The second and third sessions were divided into different phases:

Phase 1. Warm-up and pre-test DST

Always before the endurance test, the subjects did a basic warm-up based on a continuous 15-minute run and joint mobility. RPE was measured to check whether the subjects were at a similar level of pre-race fatigue.

\section{Phase 2. Performance of the $10 \mathrm{~km}$ endurance test}

The study consisted of two resistance tests of similar distance $(10 \mathrm{~km})(\mathrm{TR} 10)$ and intensity in two environments, artificial (TR10A) and natural (TR10N). To confirm that the results of the test were not affected by the intensity, two mainly flat circuits with a similar accumulated difference in the level were selected (natural $=87$ meters and artificial $=56$ meters). In addition, it was confirmed with GPS that the race pace was between $4.00 \mathrm{~min} / \mathrm{km}$ and $4.10 \mathrm{~min} / \mathrm{km}$. The subjective perception of effort (RPE) was used both before and after the race, as well as after a five-minute rest period. The pavement of both circuits was also controlled, trying to run on a stable surface free of considerable obstacles such as cobbles, sand, or any other factor that could influence performance.

The measurement of the resistance tests was performed alone on different days and times for each of the subjects. The order in which the tests were carried out was randomized, leaving one week between the 10 $\mathrm{km}$ race in an urban environment and the one in a natural environment.

Phase 3. Post-test DSP in two stages

Just at the end of the TR10A and TR10N, the subjects filled in the relevant RPE and were given the Digit Span Test-backward (DST). This process was repeated after a five-minute break. The protocol for performing the DST was carried out in accordance with standard administration. The test was applied to each of the environments on three occasions: before, after, and after five minutes of TR10. A randomized number sequence was read, with one number being spoken every second and increasing from 2 to 8 . Subjects had to repeat the number sequence backward. If an error occurred in a number sequence, the previous number sequence that had been able to repeat correctly was recorded as a score.

\section{Instruments}

The Digit Span Test backward (DST) for the evaluation of working memory and attention was included and applied in the study. The DST protocol was carried out according to the standard administration, using a tablet to carry out the data recording. The subjective perception of effort (RPE) was evaluated before and after performing the DST for each of the environments. And finally, GPS was used to control the race pace. 


\section{Statistical analyses}

Descriptive statistics are represented as mean (SD). Tests of normal distribution and homogeneity (Kolmogorov-Smirnov and Levene's, respectively) were conducted on all data before analysis. Wilcoxon test was used for determining within-group differences as a repeated measure analysis (pre-post). Analyses of one-way ANOVAs were used to analyse the Digit Span Test. A t-tests were used to compared Physiological parameters (RPE). Statistically significant effects were further analysed by paired-sample t-tests [0.2 (small); 0.5 (medium) and > 0.8 (large)] corrected by Holm-Bonferroni for multiple comparisons. Effect size is indicated with Cohen's d for t-tests. Data were analysed using software Statistica (version 10.0; Statsoft, Inc., Tulsa, OK, USA). For all analyses, significance was accepted at $p<.05$. All data were transformed to 10 logarithmic scale.

\section{RESULTS}

\section{Physiological parameters (RPE)}

A paired sample t-test with RPE [Pre-PEN Condition $(0.17 \pm 0.16)$ and Pre-PEA $(0.23 \pm 0.16)$ ] was no significant. However, a t-test with RPE [Pre-PEN Condition $(0.17 \pm 0.16)$ and Post-PEN $(0.93 \pm 0.03)$ ] and [Pre-PEA Condition $(0.23 \pm 0.16)$ and Pre-PEA Condition $(0.94 \pm 0.03)$ ] showed higher values in both post conditions, $t(13)=15.27, p<.001, d=6.60$ and $t(13)=15.93, p<.001, d=6.16$, respectively. See Figure 1.

\section{Digit Span Test}

RTs. First, a One-way ANOVA with participants' mean DSTA (Pre-PEN Condition Vs Pre-PEA Condition), was not significant, $F<1$. Second, a one-way ANOVA with participants' mean DSTA (Pre-PEN Condition and Post-PEN Condition), also was significant, $(p>$.05). However, other one way ANOVA with Pre-PEA Condition $(0.23 \pm 0.16)$ and Post-PEA $(0.94 \pm 0.03)]$ revealed a significant main effect of environment condition, $t(13)=2.14, p<.05, d=0.62$. See Figure 1 .

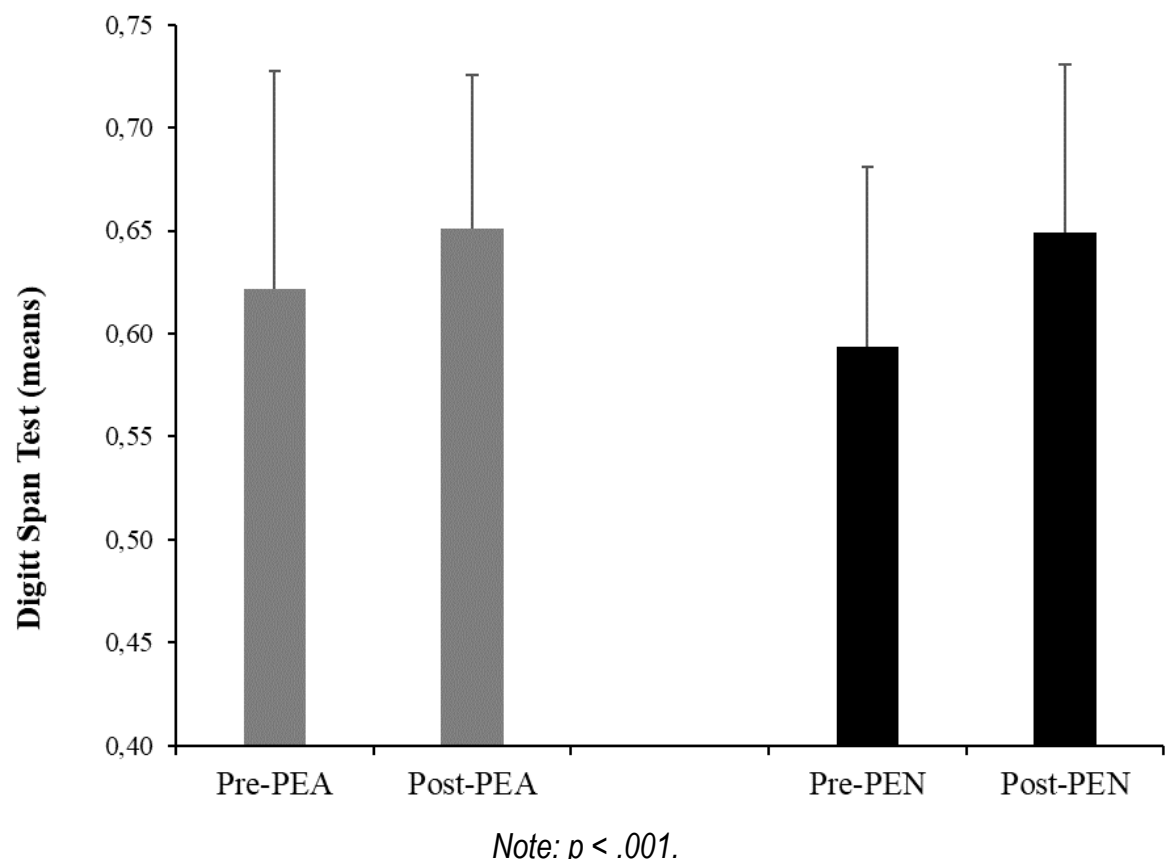

Figure 1. Physical exercises environment condition. 


\section{DISCUSSION}

The objective of this research was to compare the results obtained by highly trained University Students in a test of attention and working memory such as DST, after a physical exercise intervention in two different environmental contexts. The behaviour on the physical exercise's variables that could produce effects on cognitive performance has been the objective of the study. Specifically, the present work aimed to analyse the effect of running a 10,000 meter run at a medium-high intensity on working memory and attention in semiprofessional athletes. Our results show how performance in the DST test seems to improve significantly with the performance of a medium-high intensity aerobic exercise of approximately 40 minutes when it is performed in natural environments. Also, no relationship has been observed between DST performance and RPE. The results obtained indicate that the performance of PEA has an acute effect on working memory, suggesting that attention span and immediate verbal recall may be improved.

The binomial physical exercise and cognition seem to be highly related, with different systematic reviews and meta-analyses in the scientific literature that confirm a positive relationship (Álvarez-Bueno et al., 2017; Biddle \& Asare, 2011). However, there is a multitude of cognitive functions that must be studied independently, such as, for example, working memory, which is conceived as a function that involves the temporary storage capacity of information to be used in solving complex cognitive tasks., such as learning and reasoning or routine situations, such as remembering a phone number (Gathercole, Alloway, Willis \& Adam, 2006). The hypothesis raised in this research asserted that the results obtained in the DST test would not be modified by carrying out the physical exercise in different environments. However, the results obtained show how the TR10N intervention significantly improved the results in the DST test, which would mean rejecting the research hypothesis and accepting the null hypothesis.

The results found in this study agree with those found in antecedent research, such as those examined in people walking (Berman et al., 2008), on a cycle ergometer (Rogerson et al., 2016), and running (Rogerson \& Barton, 2015). However, the effect of a physical exercise intervention in natural environments does not always offer an improvement in DST performance compared to artificial environments (Bodin \& Hartig, 2003; Bratman et al., 2015; de Brito et al., 2019). This disagreement in the reviewed results can be explained by the diversity of methodological factors, which could cause the discrepancies found (Baena et al., 2020). These authors presented a series of characteristics that should be respected by future research aimed at comparing interventions in two settings. For example, the importance of performing the cognitive test in the same environment to isolate the effect of the intervention based on physical exercise is highlighted, also highlighting the need to control the intensity of exercise. In addition to these factors, it has been detected how studies with designs similar to the one presented have used different samples, mainly with university students, which made it difficult to confirm the existence of a similar level of physical condition. Finally, control over external stimuli that could cause marked wear and tear of selective attention is a variable that should be considered to explain the discrepancies found in the studies (Bratman et al., 2015; Kaplan, 1995). An excessive load of stimuli in both environments, such as avoiding a car or a pedestrian, as well as an excessively dense forest, can produce an alteration of the results as a consequence of complicating the restoration of the attention that is implicitly assumed to the natural environments (Kaplan, 1993; Kaplan, 1995).

Also, our results increase the theory that physical exercise in natural environments has an acute positive effect on working memory. The diversity of types of exercise, their intensity, and the study samples make it difficult for the results found to be extrapolated to other populations. Now, it can be suggested that the performance of a moderate-high intensity training by the athlete could have a positive effect on the ability to 
analyse and process information more fluently, improving attention to potential environmental demands that could offer the environment.

Finally, several limitations have been found in the study design. It seems to indicate that the result of the DST test could be influenced by the participants' cultural-academic level (Colom et al., 2007). For this reason, control over the variable of the educational level of the sample must be considered in order to confirm the acute effect of physical exercise. Furthermore, due to the small sample size, the research with a control group was not implemented. However, and despite these limitations, it should be noted that this research has proposed a similar design to previous studies that had similar objectives. Because we wanted to evaluate the acute effect of physical exercise in two environmental contexts in the same subject, it was decided not to implement a control group. Additionally, this research has been characterized by overcoming the methodological limitations found in the predecessor studies, such as the individual performance of the physical exercise, that the control of the intensity of the interventions was similar through the RPE and with GPS and that the performance evaluation of the DST test was performed in the same context to isolate the effect of the environment on performance.

\section{CONCLUSION}

In summary, it has been shown how long-duration, high-intensity exercise has an acute positive effect on performance in the Digit Span Backward test in highly trained University Students. This improvement in working memory is significant when the activity is carried out in natural environments. Likewise, the control of the intensity of the exercise through the RPE allows to confirm the influence of the environment in the results, since both contexts (artificial and natural) offered a similar difficulty for the evaluated subjects. Finally, it should be noted that the methodological design proposed in this work can serve to overcome the shortcomings found in previous research. In addition, since the results obtained cannot be extrapolated to the general population due to the peculiarity of the sample examined, it is advisable to carry out future research that modifies the intensity and duration of physical exercise to evaluate to what extent the improvement obtained is a consequence of the environment or of the physical activity carried out.

\section{REFERENCES}

Álvarez-Bueno, C., Pesce, C., Cavero-Redondo, I., Sánchez-López, M., Martínez-Hortelano, J. A., \& Martínez-Vizcaíno, V. (2017). The Effect of Physical Activity Interventions on Children's Cognition and Metacognition: A Systematic Review and Meta-Analysis. Journal of the American Academy of Child and Adolescent Psychiatry, 56(9), 729-738. https://doi.org/10.1016/j.jaac.2017.06.012

Aubert, S., Barnes, J. D., Abdeta, C., Nader, P. A., Adeniyi, A. F., Aguilar-Farias, N., Tenesaca, D. S. A., Bhawra, J., Brazo-Sayavera, J., Cardon, G., Chang, C. K., Delisle Nyström, C., Demetriou, Y., Draper, C. E., Edwards, L., Emeljanovas, A., Gába, A., Galaviz, K. I., González, S. A., ... Tremblay, M. S. (2018). Global Matrix 3.0 physical activity Report Card grades for children and youth: Results and analysis from 49 countries. Journal of Physical Activity and Health, 15(Suppl 2), S251-S273. https://doi.org/10.1123/jpah.2018-0472

Baena-Morales, S., González-Fernández, F.T García-Taibo, O., López-Morales, J, A., (In Press). Caracterización de los procesos cognitivos relacionados con la atención selectiva en entornos naturales y su relación con la actividad física. Una revisión. Sport-TK.

Bailey, A. W., Allen, G., Herndon, J., \& Demastus, C. (2018). Cognitive benefits of walking in natural versus built environments. World Leisure Journal, 60(4), 293-305. https://doi.org/10.1080/16078055.2018.1445025 
Berman, M. G., Jonides, J., \& Kaplan, S. (2008). The Cognitive Benefits of Interacting With Nature | The Center for Health Design. Psychological Science Research, 19(12), 1207-1212. https://doi.org/10.1111/j.1467-9280.2008.02225.x

Biddle, S. J. H., \& Asare, M. (2011). Physical activity and mental health in children and adolescents: A review of reviews. British Journal of Sports Medicine, 45(11), 886-895. https://doi.org/10.1136/bjsports-2011-090185

Bodin, M., \& Hartig, T. (2003). Does the outdoor environment matter for psychological restoration gained through running? Psychology of Sport and Exercise, 4(2), 141-153. https://doi.org/10.1016/S14690292(01)00038-3

Bowler, D. E., Buyung-Ali, L. M., Knight, T. M., \& Pullin, A. S. (2010). A systematic review of evidence for the added benefits to health of exposure to natural environments. BMC Public Health, 10. https://doi.org/10.1186/1471-2458-10-456

Bratman, G. N., Daily, G. C., Levy, B. J., \& Gross, J. J. (2015). The benefits of nature experience: Improved affect and cognition. Landscape and Urban Planning, 138, 41-50. https://doi.org/10.1016/j.landurbplan.2015.02.005

Bratman, G. N., Hamilton, J. P., \& Daily, G. C. (2012). The impacts of nature experience on human cognitive function and mental health. Annals of the New York Academy of Sciences, 1249(1), 118136. https://doi.org/10.1111/j.1749-6632.2011.06400.x

Coles, K., \& Tomporowski, P. D. (2008). Effects of acute exercise on executive processing, short-term and long-term memory. Journal of Sports Sciences, 26(3), 333-344. https://doi.org/10.1080/02640410701591417

Colom, R., Escorial, S., Shih, P. C., \& Privado, J. (2007). Fluid intelligence, memory span, and temperament difficulties predict academic performance of young adolescents. Personality and Individual Differences, 42(8), 1503-1514. https://doi.org/10.1016/i.paid.2006.10.023

Costigan, S. A., Eather, N., Plotnikoff, R. C., Hillman, C. H., \& Lubans, D. R. (2016). High-Intensity Interval Training for Cognitive and Mental Health in Adolescents. Medicine and Science in Sports and Exercise, 48(10), 1985-1993. https://doi.org/10.1249/MSS.0000000000000993

de Brito, J. N., Pope, Z. C., Mitchell, N. R., Schneider, I. E., Larson, J. M., Horton, T. H., \& Pereira, M. A. (2019). Changes in psychological and cognitive outcomes after green versus suburban walking: A pilot crossover study. International Journal of Environmental Research and Public Health, 16(16), 112. https://doi.org/10.3390/ijerph16162894

Fuegen, K., \& Breitenbecher, K. H. (2018). Walking and being outdoors in nature increase positive affect and energy. Ecopsychology, 10(1), 14-25. https://doi.org/10.1089/eco.2017.0036

Groth-Marnat G \& Baker S. (2003). Digit Span as a Measure of Everyday Attention: A Study of Ecological Validity. Perceptual and Motor Skills.;97:1209-1218. doi:10.2466/pms.2003.97.3f.1209

González Fernández, F. T., Baena-Morales, S., Vila Blanch, M., \& García Taibo, O. (2020). Efectos regulares en la cognición de los descansos activos. Sportis. Scientific Journal of School Sport, Physical Education and Psychomotricity, 6(3), 488-502. https://doi.org/10.17979/sportis.2020.6.3.6414

Harte, J. L., \& Eifer, G. H. (1995). The effects of running, environment, and attentional focus on athletes' catecholamine and cortisol levels and mood. Psychophysiology, 32(1), 49-54. https://doi.org/10.1111/j.1469-8986.1995.tb03405.x

Hartig, T., Evans, G. W., Jamner, L. D., Davis, D. S., \& Gärling, T. (2003). Tracking restoration in natural and urban field settings. Journal of Environmental Psychology, 23(2), 109-123. https://doi.org/10.1016/S0272-4944(02)00109-3 
Hillman, C. H., Kamijo, K., \& Scudder, M. (2011). A review of chronic and acute physical activity participation on neuroelectric measures of brain health and cognition during childhood. Preventive Medicine, 52(SUPPL.), S21-S28. https://doi.org/10.1016/j.ypmed.2011.01.024

Kaplan, R. (1993). The role of nature in the context of the workplace. Landscape and Urban Planning, 26(1-4), 193-201. https://doi.org/10.1016/0169-2046(93)90016-7

Kaplan, S. (1995). The restorative benefits of nature: Toward an integrative framework. Journal of Environmental Psychology, 15(3), 169-182. https://doi.org/10.1016/0272-4944(95)90001-2

Lahart, I., Darcy, P., Gidlow, C., \& Calogiuri, G. (2019). The effects of green exercise on physical and mental wellbeing: A systematic review. International Journal of Environmental Research and Public Health, 16(8). https://doi.org/10.3390/ijerph16081352

Lambourne, K., \& Tomporowski, P. (2010). The effect of exercise-induced arousal on cognitive task performance: A meta-regression analysis. Brain Research, 1341, 12-24. https://doi.org/10.1016/j.brainres.2010.03.091

Lee, A. C. K., \& Maheswaran, R. (2011). The health benefits of urban green spaces: A review of the evidence. Journal of Public Health, 33(2), 212-222. https://doi.org/10.1093/pubmed/fdq068

Naveen, Mishra, T., Tiwari, T., Singh, I. L., Singh, A. L., \& Singh, T. (2017). Effect of aerobic exercise on reaction time during vigilance task performance. Journal of the Indian Academy of Applied Psychology, 43(2), 205-210.

Ostrosky-Solís \& Lozano A. (2006) Digit Span: Effect of education and culture, International Journal of Psychology, 41:5, 333-341. https://doi.org/10.1080/00207590500345724

Pretty, J. (2004). How nature contributes to mental and physical health. Spirituality and Health International, 5(2), 68-78. https://doi.org/10.1002/shi.220

Rogerson, M., \& Barton, J. L. (2015). Effects of the Visual Exercise Environments on Cognitive Directed Attention, Energy Expenditure and Perceived Exertion. International Journal of Environmental Research and Public Health, 16(23), 7321-7336. https://doi.org/10.3390/ijerph120707321

Rogerson, M., Gladwell, V. F., Gallagher, D. J., \& Barton, J. L. (2016). Influences of green outdoors versus indoors environmental settings on psychological and social outcomes of controlled exercise. International Journal of Environmental Research and Public Health, 13(4). https://doi.org/10.3390/ijerph13040363

Sanabria, D., Luque-Casado, A., Perales, J. C., Ballester, R., Ciria, L. F., Huertas, F., \& Perakakis, P. (2019). The relationship between vigilance capacity and physical exercise: A mixed-effects multistudy analysis. PeerJ, 2019(6), 1-15. https://doi.org/10.7717/peeri.7118

Singh, A. S., Saliasi, E., Van Den Berg, V., Uijtdewilligen, L., De Groot, R. H. M., Jolles, J., Andersen, L. B., Bailey, R., Chang, Y. K., Diamond, A., Ericsson, I., Etnier, J. L., Fedewa, A. L., Hillman, C. H., McMorris, T., Pesce, C., Pühse, U., Tomporowski, P. D., \& Chinapaw, M. J. M. (2019). Effects of physical activity interventions on cognitive and academic performance in children and adolescents: A novel combination of a systematic review and recommendations from an expert panel. British Journal of Sports Medicine, 53(10), 640-647. https://doi.org/10.1136/bjsports-2017-098136

Thompson Coon, J., Boddy, K., Stein, K., Whear, R., Barton, J., \& Depledge, M. H. (2011). Does participating in physical activity in outdoor natural environments have a greater effect on physical and mental wellbeing than physical activity indoors? A systematic review. Environmental Science and Technology, 45(5), 1761-1772. https://doi.org/10.1021/es102947t

Tomporowski, P. D., \& Ganio, M. S. (2006). Short-term effects of aerobic exercise on executive processing, memory, and emotional reactivity. International Journal of Sport and Exercise Psychology, 4(1), 57-72. https://doi.org/10.1080/1612197x.2006.9671784 


\section{(c) $)(1) \Theta(9)$}

This work is licensed under a Attribution-NonCommercial-NoDerivatives 4.0 International (CC BY-NC-ND 4.0). 